\title{
A Study into Autonomous Scanning for 3D Model Construction
}

\author{
Darryl O’Hare, William Hurst, David Tully, Abdennour El Rhalibi \\ Department of Computer Science, \\ Liverpool John Moores University, \\ Byrom Street \\ Liverpool, L3 3AF, UK \\ D.OHare@2013.ljmu.ac.uk, \{W.Hurst, D.Tully, \\ A.Elrhalibi\}@ljmu.ac.uk
}

\begin{abstract}
D scanning and printing has the potential to revolutionise the world. It offers a bridge between the virtual environment and the tangible world. The use of $3 \mathrm{D}$ scanners to capture and recreate defining objects is known as $3 \mathrm{D}$ virtualisation. It involves capturing a real-life scene using laser technology and representing its geometry using 3D modelling software or 3D printers. Despite being a relatively young technology, 3D printing has now become accessible and a part of modern industry. The printing of a 3D generated model can change the way in which an individual understands a concept, environment or communicates an idea. This has multiple benefits, for education, skills development, training and within the construction industry. However, using this technology relies on the operator having the skills and training required to generate accurate 3D models, and account for errors in the mesh after scanning. As such, this paper details the development into an automated 3D scanning system, and a cloud-based printing platform, where models are intelligently printed by multiple devices. Its development allows the readiness of 3D printing capabilities to unskilled users, who have no education or training in 3D model construction. Objects can be instantly manipulated and transferred into free-to-use open source graphic software. The access to detailed 3D model construction has never been so accessible to the untrained.
\end{abstract}

Keywords: 3D Scanning; 3D Modelling; Education; Real-Time

\section{Introduction}

3D printing has become a common tool for developing bespoke applications in construction, engineering, and computing industries. It is, however, a relatively young technology and the full applicability of 3D printing is still being investigated. One of the common prohibitive issues in 3D printing is its exclusive reliance on designers having adequate skills to create, edit and design 3D models suitable for 3D printing (Latham, $\mathrm{K}$, et al.,). The creation of printable 3D models requires specialised knowledge of 3D editing software. At present, this does not favour consumers of 3D printing technology

adfa, p. 1, 2011.

(C) Springer-Verlag Berlin Heidelberg 2011 
who have no 3D modelling capabilities. Therefore, model creation requires a steep learning curve and additional expenditure in relation to $3 \mathrm{D}$ modelling, rendering and animation software.

Yet, architects or construction workers tend to make use of 3D construction models to plan out development and renovations. However, in practical environments, this requires the expertise of 3D modellers to construct and map out rooms or buildings inside or out in relevant 3D software. In addition, the use of off-the-shelf 3D printing technology involves techniques, which are generally slow and time-consuming.

Therefore, this project involves the development of an autonomous system for 3D room construction, object cloning and bespoke object construction. With current IoT-ready devices, it is possible to scan and image capture environments for 3D printing purposes. By autonomously converting basic measurements or images into a 3D virtual scene, an automated system would be able to 3D-clone structures or models of people etc. Additive manufacturing using a cloud-based multi-agent system can provide an approach to improve the speed of 3D printing technology (Van der Hoek et al.,). As such, we envision that intelligent agents can be employed to divide up 3D model structures, and distribute the segments to an arrangement of 3D printers. A self-managed 3D printing service, using a cloud-based model, is a relatively untried research area.

As a final objective, we will investigate how this new technology can be used by both individuals and higher education establishments to improve the way, in which, technical and complex subjects can be taught. Current 3D technologies enable a more indepth platform for communicating information (Keskin et al.,). For education and teaching computer/complex theoretical concepts, adding a tangible component may change the way in which a subject is communicated (I. Papp, R et al.,). This paper is organised as follow. Section 2 presents a background overview on 3D model construction and 3D scanning techniques. Section 3 presents our system framework and design for an autonomous 3D scanning device. Section 4 presents an evaluation and discussion on the approach put forward. The future work and conclusion are presented in Section 5 .

\section{Background}

When viewing a real-world tangible object, there are various aspects which require consideration. Firstly, as the reference object provides the main information for the 3D model construction, measurements need to be accurate in order to represent the realworld object as a digital output. This is a time-consuming and sometimes a costly process. Creating 3D scans using digital image capture technologies produces instant 3D rendered geometry, which is an accurate recreating of the real world object. The process is often as rudimentary as taking a series of photos or recording a video with a handheld device. Recreating an object using a camera and depth scanner has traditionally been a challenging task. However, the reality is that the post-production of a 3D scan is usually the most time consuming aspect of creating a digital representation. It is often determined by many factors, such as the GPU or CPU, which both determine the capture frame rates, and how well the hardware and the software working together actually are. The scanning process is still a task worthy enterprise and can be time consuming 
in its own way but this paper looks at different technologies and how this method can be improved upon making it a viable system for geometry creation. As such, in the section, related research on $3 \mathrm{D}$ scanning and modelling is presented.

\subsection{Scanning Types}

The Microsoft Kinect has a built in sensor and a high resolution RGB (Red, Green, Blue) camera capable of depth capture and 3D Mesh geometry creation (Nagori et al.,). Over the last 8 years Microsoft have invested significant amounts of money and research in 3D scanning and print options; the Hololens is testament to this (Microsoft et $a l$.,). They have made available free Kinect-based software to make it a viable option for users to scan an object and print out the results using a 3D printer. With the software, it is possible to create novel algorithms for motion or image capture.

In the construction industry, full-scale models can be 3D printed out from scanners attached to drones used to map out an entire cave; before any human life is put at risk venturing down (Ende et al.,). This now affordable technology is changing the whole perception of 3D model construction in industry. Whereas, traditionally scanning an object, or getting a general shape, primitive techniques involved using Radar. This involves using a radio wave signal, projected out and used to shape-map an area by calculating the points where the signal would hit the object and reflect the signal back at the receiver.

More modern revolutionary techniques are found in the form of LiDAR (Tully(a), et $a l$. .). Where instead of radio frequencies, a strong pulse of light is sent out and, by understanding the amount of time light takes to hit a surface and reflect back, a distance can be calculated precisely. This enables an engineer to determine the $\mathrm{X}, \mathrm{Y}$ and $\mathrm{Z}$ coordinates of an object, which would then be used for a mapping sequence. After the first return, sequential pulses begin to create an image linking up and creating a geometry based on each coordinate created from the scanner. This would then be stored in a library of points to create a full point cloud. This is the high-end of the laser scanning spectrum, often used by geologists and meteorologists. However, LiDAR uses different laser types for various projects and conditions. Off-the-shelf scanners, like the Kinect, use a similar approach, where a scanner uses LiDAR to calculate depth.

With regards to LiDAR data, when mapping large scale environments, there are issues which may affect its accuracy. LiDAR has a high cost and, city LiDAR maps are not always up-to-date in ever changing urban environments. There is often missing data, caused by vegetation or water for which the laser used does not reflect off the object correctly. False data can also be captured from dynamic objects; vehicles, flocks of birds, swaying trees etc.

As Russel et al., state, LiDAR produces improved results of data capture in daylight hours. The issue with this is if capturing in daytime hours, multiple moving artefacts are more frequently found within the data-source. The resolution of the LiDAR maps proves troublesome in respect to processing and also data storage. Each resolution and data point count contained within each map is stated below in Table 1. 
Tab. 1 LiDAR data resolutions, dimensions, point count, and area coverage.

\begin{tabular}{|c|c|c|c|}
\hline Resolution & Dimensions & Total points contained & $\begin{array}{c}\text { Area Cov- } \\
\text { erage }\end{array}$ \\
\hline $\mathbf{2 m}$ & $500^{2}$ & 250,000 & $1 \mathrm{~km}^{2}$ \\
\hline $\mathbf{1 m}$ & 1000 & $1,000,000$ & $1 \mathrm{~km}^{2}$ \\
\hline $\mathbf{5 0 c m}$ & $1000^{2}$ & $1,000,000$ & $500 \mathrm{~m}^{2}$ \\
\hline $\mathbf{2 5} \mathbf{c m}$ & $2000^{2}$ & $4,000,000$ & $500 \mathrm{~m}^{2}$ \\
\hline
\end{tabular}

The military already use LiDAR point technology in automation and have also created robots that can scan an entire building before any soldier even enters the vicinity, always helping to reduce the loss of life. Figure 1 below, is an example of a LiDAR generated map.

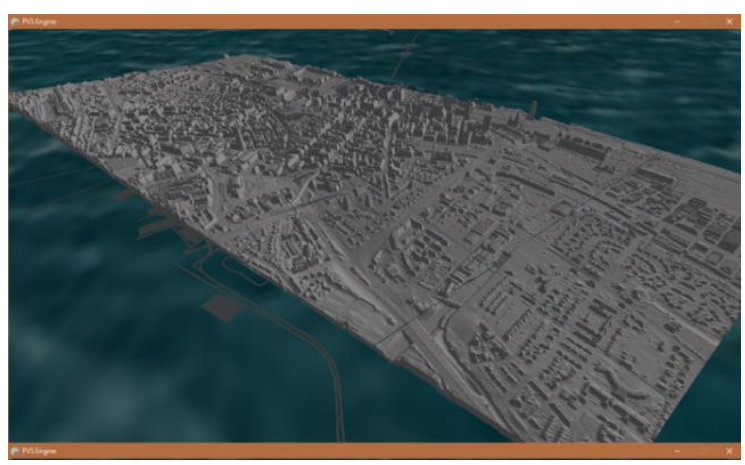

Fig. 1. LiDAR Generated Map

The modern world needs to reproduce things at a rate that humans cannot keep up with. 'Time of Flight' cameras are relatively new, in 3D scanning domain, but within photogrammetry the technique has been around for decades (Anand et al.,). The process works at a high frame rate and in a similar method to the LiDAR depth camera technology, where it measures the time of flight between a light signal from the camera to the object and then measures all the points it has projected onto the object. This approach is typically used in the construction industry for measuring distances between objects, measuring rooms, and also measuring the size and shape of an object.

\section{$2.23 D$ and VR}

Scanned 3D objects are typically deployed into virtual reality (VR) systems. VR involves user interactions between digital data in a representation of the real world (Latham, K, et al.,). Often, this means the user is completely immersed in an artificial environment through use of a headset, or interacts through the environment on a screen. However creating an emulated environment is a complex procedure when modelling by hand. To develop virtualised environments, a skilled developer must create objects, as well as procedurally generated buildings, by hand or by use of algorithms (LiangKun et al.,). Yet, the use of 3D environments for education and learning is becoming increasingly commonplace. As Zhang et al., discuss, the use of a 3D virtual cloud plat- 
form can be employed for language education. As detailed in their research, the development of their system requires extensive code development and the modelling of a 3D 'game room' for the users to interact with (Zhang et al). Approaches such as this, where there is crossover between 3D modelling and games technology for real-world simulation, is becoming widespread (Tully(b), et al.,) as an effective way of learning and communicating. This process is known as Game Based Learning and it's trend in recent years has been notable in pedagogic research theories (H. Chen et al., ). As such, the research presented in the paper, aims to make 3D content construction more available to the unskilled user for virtual environment creation, or 3D model construction; both for educational purposes.

\section{Approach}

The system put forward in this paper theorises that objects and environments can be autonomously scanned using off-the-shelf technologies and the models subsequently made accessible to the untrained. The system has a modular design, where a cloudbased printing platform is employed to intelligently reconstruct accurate scale representations of the 3D scanned object. In this section, an overview of the system design is presented along with the initial experimentation. The full purpose of the system is to scan a Tangible object in real time, process the scan and output a final lower polygon mesh suitable for $3 \mathrm{D}$ environments as discussed above.

\subsection{System Design}

Figure 2 displays a high-level view of the system design. The process is completed in 4 stages which are outlined.

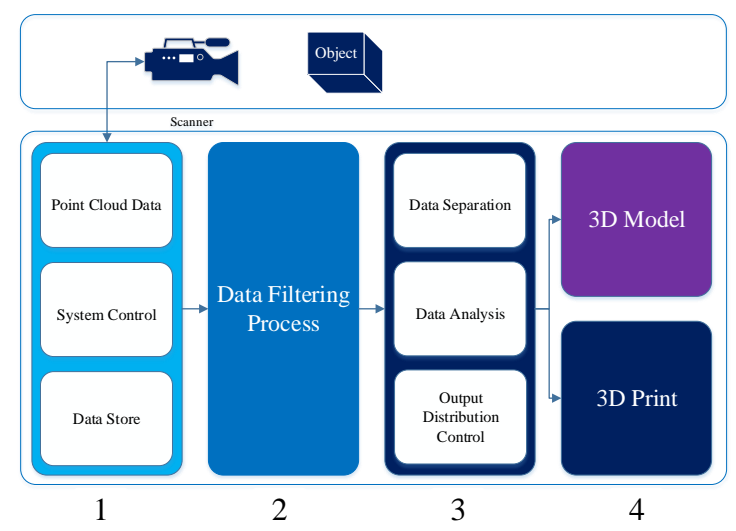

Fig. 2. System Framework and Process

For the scanning process, nearly all 3D scanners use a point cloud system to map and generate a shape. Each vertex created within the point cloud has its own $X, Y, Z$ location. Combined, all these coordinates represent an entire model. Polygons can then be created from these coordinates in a $3 \mathrm{D}$ space. The positioning can be manipulated post 
process but the 3D scanner creates accurate values for each vertex and full mesh geometry can be instantly created. After the vertex locations are complete and indexed, most scanning software completes a rasterisation process, turning all the newly created geometric shapes into a format capable of being seen in a renderer. At this stage textures can be applied and the model can really take shape.

To make the desired geometry of a mesh for optimizing purposes, the topology is considered. The topology is the general flow of edges, vertices and polygons of an object. Mesh optimising, creates a topology, can be crucial for frame rates and rendering, making a mesh or animation move in an environment accurately. As such, the system processes are explained as follows:

1. Object scanning is governed by the system control module, which controls the intake of point cloud data. The collected data is then placed in a temporary database.

2. The data is extracted from the temporary databased where it is filtered to check for missing values.

3. Next, in stage three, the object is divided into segments. For example, in the case of a human as the scanned object, the arms, legs, body and head would be separated and treated as different objects.

4. The model is then either sent for reconstruction in a 3D software application or printed out on a 3D cloud-based printing platform.

\subsection{Case Study}

To demonstrate the approach of our system, a simple scan was made of an individual participant using the Kinect v2 with minimum clothing and only scanning from the waist up. The scanning process is automated, using the system discussed in Section 3.1.
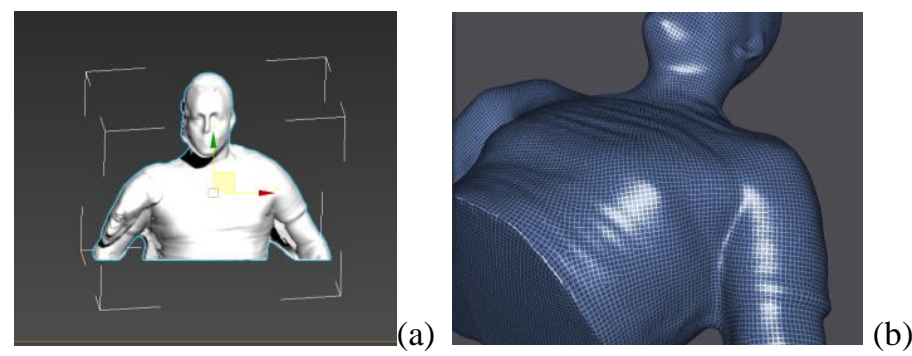

Fig. 3. 3D Scanned Person(a) and Object after Reconstruction (b)

As the scanner employed is a Kinect v2, it meant that the polygon count was considerably high at 320,000 polygons, due to the point cloud mesh generation. This is why the third stage in the system design works to Retoplogize the new object shown in Figure 3 (b) by creating a flow of quadrilateral polygons for considerably lowering the vertices count. In Figure 4(a), the first model is the full mesh and in (b) the texture map applies perfectly onto each triangle, as it has its own UV mapped coordinates. However once the mesh has been quadifed, the UV Map coordinates are then moved around with the new mesh geometry. 

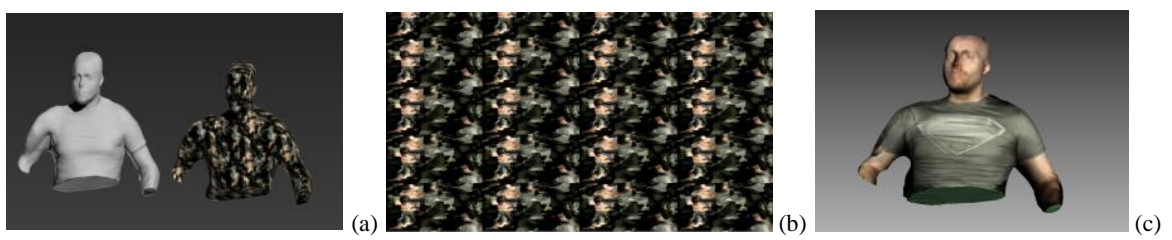

Fig. 4. Full Mesh and lowered Mesh(a), Texture Map (b), Lowered Polygon Model with baked textures(c)

Currently, dissecting the generated UV map at this point would be a considerable challenge for a 3D processing software. So a new texture mapping approach needs to be administered to the system. This process, known as 'Baking', takes the two meshes, side by side, the original mesh 1 and the new quadifed lowered mesh 2 . The technique is used to transfer details from one object to another. It functions by scanning surface detail of the first object and saving the information as a texture map, using the first model's texture coordinates. The baking process takes place via the Computer Graphics software. All the texture details from mesh 1 are cast onto mesh 2. In our case study, we cast our textures onto the new lowered qualified mesh, generating a new UV Map in the process.

\subsection{Output}

The final output model shown in Figure 5 demonstrates a completed modified mesh. To complete the final output for use in real time rendering applications the mesh needs to be closed. An approach would be to employ a 'cap-hole modifier', within 3D software. This would fill any holes in a mesh and remove unnecessary triangles. This however, would require training in 3D software so would not be ideal for the approach put forward. After use of a cap-hole modifier, the final output is presented in Figure 5 and is comprised of 37,000 polygons showing a good rational of the system with $10 \%$ of the original poly count remaining with no degradation in the mesh.

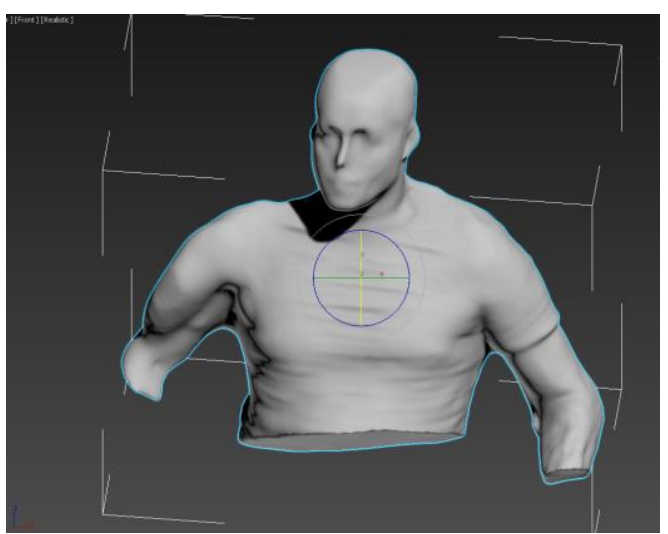

Fig. 5. Final Output 


\section{Conclusion and Future Work}

Digital scanning technology is becoming common place in education and in many industries. The use of $3 \mathrm{D}$ technologies can transform the way in which a concept is understood. With the increase in available off-the-shelf 3D scanning technologies such as the Kinect, 3D modelling and object reconstruction for the untrained has never before been so accessible. As such, the system presented in the paper aims to make 3D modelling more accessible to untrained users, through use of an automated scanning and modelling approach. In our future research, we will work towards the integration of artificial intelligence to understand the type of 3D object being scanned and divide the object into different parts so they can be treated as separate objects. This mean that, high detail can be captured from the correct parts and low details from the unimportant parts to ensure that file sizes are kept to a minimum.

\section{$5 \quad$ References}

1. Anand, C., Jainwal, K., and Sarkar, M., A high background light subtraction circuit for long range time-of-flight cameras, 2016 IEEE Asia Pacific Conference on Circuits and Systems (APCCAS), Jeju, pp. 487-490, 2016

2. Chen, J. H., Shih, T. K., and Chen, J. Y., To develop the ubiquitous adventure RPG (role play game) game-based learning system, IEEE International Conference on Systems, Man, and Cybernetics (SMC), Seoul, 2012, pp. 2973-2978.

3. Ende, B. A. am., 3D mapping of underwater caves, in IEEE Computer Graphics and Applications, vol. 21, no. 2, pp. 14-20, Mar/Apr 2001

4. Keskin, C., Balci, K., Aran, O., Sankur, B., and Akarun, L.,A Multimodal 3D Healthcare Communication System, 3DTV Conference, Kos Island, 2007, pp. 1-4.

5. Latham, K., Hurst, W., Shone, N., El Rhalibi, A., and Pan, Z., A Case Study on the Advantages of 3D Walkthroughs over Photo Stitching Techniques, Submitted to The International Conference on Virtual Reality and Visualization (ICVRV 2016), China

6. Microsoft Hololens Tutorial, 2016 IEEE International Symposium on Mixed and Augmented Reality (ISMAR-Adjunct), Merida, 2016.

7. Nagori, N. P., and Malode, V., Communication Interface for Deaf-Mute People using Microsoft Kinect, International Conference on Automatic Control and Dynamic Optimization Techniques (ICACDOT), Pune, India, pp. 640-644, 2016

8. Papp, I., Tornai, R., and Zichar, M., What 3D technologies can bring to education: The impacts of acquiring a 3D printer, 7th IEEE International Conference on Cognitive Infocommunications (CogInfoCom), Wroclaw, pp. 000257-000262, 2016.

9. Russel S, Artificial Intelligence: A Modern Approach, 3rd Edition. Prentice Hill, 2009.

10. LiangKun, $S$ and DeTong, Z., Polygon topology generation algorithm research based on $3 \mathrm{~d}$ modeling, IEEE 2nd International Conference on Software Engineering and Service Science, Beijing, pp. 385-388, 2011.

11. Tully(a), D, El Rhalibi A., Pan Z., Carter C., Sudirman S, Automated Procedural Generation of Urban Environments Using Open Data for City Visualisation. International Conference on Image and Graphics. Springer International Publishing, 2015.

12. Tully(b) D., El Rhalibi A., Pan Z., Carter C., Sudirman S. (2015) Mesh Extraction from a Regular Grid Structure Using Adjacency Matrix. In: Zhang YJ. (eds) Image and Graphics. 2015. Lecture Notes in Computer Science, vol 9219. Springer, Cham 
13. Van der Hoek, W. and Wooldridge, M., 2008. Multi-agent sys-tems. Foundations of Artificial Intelligence, 3, pp.887-928.

14. Zhang B., Playing 3D: Digital technologies and novel 3d virtual environments to support the needs of Chinese learners in western education: Cross-cultural collaboration, gamification, well-being and social inclusion, 2016 22nd International Conference on Virtual System \& Multimedia (VSMM), Kuala Lumpur, Malaysia, pp. 1-9, 2016. 